\title{
Diagnosis and Management of Sciatic Endometriosis at the Greater sciatic Foramen: a Case Report
}

\author{
Robert J. $\operatorname{Trager}^{1}$ (D) Sarah E. Prosak ${ }^{1} \cdot$ Kellee A. Leonard ${ }^{1} \cdot$ Jessica E. Sigel ${ }^{2} \cdot$ Jeffery A. Dusek $^{1}$
}

Accepted: 26 April 2021 / Published online: 5 May 2021

(C) The Author(s) 2021

\begin{abstract}
Sciatic endometriosis is a rare condition in which endometrial tissue invades or compresses the lumbosacral plexus and/or sciatic nerve and causes sciatic pain, which is often cyclical. Its diagnosis depends on the recognition of signs and symptoms atypical to common degenerative lumbar disorders and its treatment requires timely and coordinated care. A 26-year-old woman presented to a chiropractor at a hospital-based outpatient clinic with a 6-month exacerbation of radiating pain and paresthesia from the right gluteal region into the leg and foot. She was previously treated for over 3 months for suspected lumbosacral radiculopathy with physical therapy. Multisegmental neurologic deficits inconsistent with her prior lumbar imaging prompted further investigation. Pelvis and hip MRI identified a $7 \times 5 \mathrm{~cm}$ mass extending through the greater sciatic foramen which compressed and infiltrated the lumbosacral plexus, sciatic nerve, and superior gluteal nerve, and was confirmed via biopsy to be endometriosis. Referral and comanagement with a neurologist and obstetrician resulted in a conservative strategy of hormone therapy with the option of having surgical excision if needed. Sciatic endometriosis is a rare disorder that may be misdiagnosed due to its tendency to mimic common lumbar disorders. This case highlights the role of a chiropractor coordinating care for a complex disorder that benefits from early diagnosis for optimal management. It also illustrates how integration of chiropractors into a hospital system can facilitate their ability to manage neuro-musculoskeletal disorders as they can easily refer to and communicate with other medical specialties within the network.
\end{abstract}

Keywords Sciatic Nerve $\cdot$ Endometriosis $\cdot$ Lumbosacral Plexus $\cdot$ Endometrioma $\cdot$ Chiropractic $\cdot$ Neurology

\section{Background}

Sciatic endometriosis describes the presence of endometrial tissue along the lumbosacral plexus and/or sciatic nerve, and is considered extrapelvic when occurring external to the pelvic cavity [1, 2]. A review published in 2019 identified 40 cases of extrapelvic sciatic endometriosis [1]. The typical presentation is a woman of reproductive age presenting with a sudden-

This article is part of the Topical Collection on Medicine

Robert J. Trager

Robert.Trager@UHhospitals.org

1 Connor Integrative Health Network, University Hospitals Cleveland Medical Center, 11100 Euclid Ave, Cleveland, OH 44106, USA

2 Department of Pathology, Case Western Reserve University School of Medicine, University Hospitals Cleveland Medical Center, 11100 Euclid Ave, Cleveland, OH 44106, USA onset, worsening, and cyclical pattern of sciatica [1]. The term "catamenial sciatica" describes the relationship of symptoms to menses [3-5].

Those with sciatic endometriosis often experience delay in diagnosis, which takes on average 3.7 years [6], at an average age of 36.6 [1]. One explanation is that sciatic endometriosis mimics the presentation of lumbar disc herniation $[4,7,6]$. Another pitfall is that only a minority of patients have typical endometriosis symptoms such as dysmenorrhea, dyspareunia, and metrorrhagia [1]. Unnecessary spinal surgeries were more common in these patients before the advent of advanced imaging $[4,6]$, but still occur [7].

Disorders affecting the lumbosacral plexus are often misdiagnosed as they are uncommon and their symptoms mimic those of lumbosacral radiculopathy [8]. One study found that disorders affecting the lumbosacral plexus and/or gluteal region contributed to symptoms in $6 \%$ of cases of sciatic pain; however, these conditions were not visualized by a standard lumbar MRI series [9]. Clinical features suggestive of a lumbosacral plexus disorder include signs and 
symptoms spanning multiple nerve root levels, absence of significant low back pain, and absence of pain with coughing/straining [8]. Special imaging techniques such as magnetic resonance neurography or coronal short tau inversion recovery (STIR) [9] or electrodiagnostic evaluations [8] can be helpful in their diagnosis.

To our knowledge, this is the first published case of extrapelvic sciatic endometriosis presenting to a chiropractor. It also may be the first to show direct magnetic resonance imaging (MRI) evidence of superior gluteal nerve entrapment by endometriosis. In prior studies, authors noted involvement of this nerve by observing the muscle denervation pattern or direct intraoperative visualization $[4,10,11]$. This case highlights the need for providers of conservative musculoskeletal care to acknowledge atypical sources of sciatic pain and be able to refer to appropriate medical specialists for continued management.

\section{Case presentation}

An otherwise healthy 26-year-old nulligravid woman, who worked as a nurse, presented to a chiropractor in an outpatient hospital-based clinic with right gluteal pain, with radiation and paresthesia into the ipsilateral leg and foot. Symptoms were present for 1 year, but suddenly worsened 6 months prior after an incident pushing a wheelchair. Pain was described as a "vice grip" around the hip and thigh, which would "come and go weeks at a time," and was slightly alleviated with ibuprofen. The patient denied having low back pain and symptoms were unaffected by coughing/straining. Her Oswestry Disability Index was 24\% (moderate disability). She had an intrauterine device (Kyleena ${ }^{\circledR}$ levonorgestrelreleasing intrauterine system) for the past year and prior to that had an etonogestrel/ethinyl estradiol vaginal ring for 5 years. Her past medical history included an asymptomatic type I Arnold-Chiari malformation. Her family history was negative for neurological and spinal disorders, and positive for osteoarthritis of peripheral joints (parents), breast cancer (paternal grandmother), and pancreatic cancer (paternal grandfather). She was previously evaluated by two physicians and completed 3 months of physical therapy for suspected lumbar radiculopathy (Fig. 1). Recent lumbar radiographs were interpreted as normal while an MRI showed a mild posterior disc bulge (Fig. 2).

Examination revealed external rotation of the right lower extremity during gait and limited right hip range of motion, with pain and spasms induced by combined flexion, adduction, internal rotation (FADIR test), and passive hip internal rotation (Frieberg's sign). Neurologic deficits were identified in the right lower extremity and included an absent Achilles reflex, $3 / 5$ strength of the extensor hallucis longus, $4 / 5$ plantar and dorsiflexion, 4/5 hip flexion (Medical Research Council grading), and reduced sensation to light touch on the foot dorsum.

The patient's presentation appeared inconsistent with radicular sciatica due to the relatively normal lumbar imaging, and presence of symptoms and deficits spanning the L4, L5, and $\mathrm{S} 1$ segments. The chiropractor ordered radiographs of the right hip and pelvis at the first office visit, which were normal, and then proceeded to MRI. The MRI revealed intramuscular edema and atrophy of the piriformis, gluteus muscles, quadratus femoris, and obturator internus, a spiculated $7 \times 5 \mathrm{~cm}$ mass extending from the sacrum through the sciatic notch with a heterogeneous T2-weighted signal, and hyperintensity and enlargement of the sciatic nerve (Figs. 3 and 4).

After discussing the images with the radiologist, the chiropractor then ordered a computed tomography (CT) scan due to suspicion of an infiltrative lesion and signs of blood and calcification. Also at this time, the chiropractor made an internal referral to an orthopedic oncologic surgeon which was facilitated by a detailed referral order and "carbon copy" of the patient's notes using a shared electronic medical records (EMR) system. Because the oncologist believed that the imaging was not suspicious for neoplasm, a neurologist was additionally consulted, who performed electromyography (EMG) and nerve conduction velocity (NCV) studies.

Electromyography showed signs of active denervation including fibrillation potentials and positive sharp waves in the extensor hallucis longus, tibialis anterior, flexor digitorum longus, tensor fascia latae, and gluteus medius. These muscles and the gluteus maximus had large, polyphasic motor units with markedly decreased recruitment. The right sural NCV was normal but reduced relative to the left side, while the right superficial peroneal sensory response was absent. The EMG and NCV findings were consistent with a lumbosacral plexopathy affecting the L5 and S1 segments. The neurologist then ordered a pelvis/hip MRI with intravenous gadolinium. This demonstrated enhancement of the sciatic foramen mass, and enhancement and enlargement of the lumbosacral trunk, S1 nerve root, and superior gluteal nerve (Fig. 5).

A CT-guided core needle biopsy was performed at the posterolateral border of the sciatic notch. Histopathological analysis (Fig. 6) showed endometrial glands and stroma with hemorrhage and hemosiderin-laden macrophages consistent with endometriosis. Additional findings included skeletal muscle atrophy and perivascular and perineural lymphoplasmacytic inflammation.

Following the diagnosis of extrapelvic sciatic endometriosis, the patient was referred to her obstetrician. The patient had a relatively early menarche at age 11 but denied dysmenorrhea, metrorrhagia, dyschezia and dyspareunia, and had no gynecologic comorbidities or infections. Transvaginal ultrasound did not reveal any intraperitoneal endometriosis. Sciatic endometriosis was managed by the gonadotropin-releasing 
Fig. 1 Timeline of events. Primary care physician (PCP), patient $(\mathrm{Pt})$, emergency department (ED), magnetic resonance imaging (MRI), physical therapy (PT), computed tomography $(\mathrm{CT})$, obstetrician (OB), endometriosis (EM). The red boxes on the left side follow the patient's management prior to presenting to our clinic (green boxes on right). Timeline created according to Scientific Writing in Health and Medicine (SWIHM) and CARE guidelines

\begin{tabular}{|l|}
\hline $\begin{array}{l}\text { An otherwise healthy 26-year old woman with a past } \\
\text { medical history of Arnold-Chiari malformation and current } \\
\text { use of an intrauterine device }\end{array}$ \\
\hline
\end{tabular}

$\mathrm{PCP}$ visit: Pt reports radiating pain into $\mathrm{R}$ leg Pt declines PT referral

\begin{tabular}{l}
\hline PCP prescribes muscle relaxant. Pt tries massage \\
Pt starts PT. Also presents to ED after a flare-up \\
Normal lumbar radiograph
\end{tabular}

Lumbar MRI

6/2020: The patient notes improvement through 2-3

months of conservative treatment and will be monitored

hormone agonist Lupron® (leuprolide acetate, $11.25 \mathrm{mg}$ every three months) and norethindrone ( $5 \mathrm{mg}$ tablet orally each day), a form of progestin, to suppress the endometrial tissue.
The patient continued to use her intrauterine device as a contraceptive as well as to provide additional treatment for endometriosis. She continued ibuprofen as needed for pain.

Fig. 2 Lumbar MRI including T2-weighted a midline sagittal image and $\mathbf{b}$ corresponding axial image of the L5-S1 intervertebral disc. Although this was interpreted as showing a mild disc bulge, there was no neural compression, and no significant findings that could be correlated with the patient's signs and symptoms
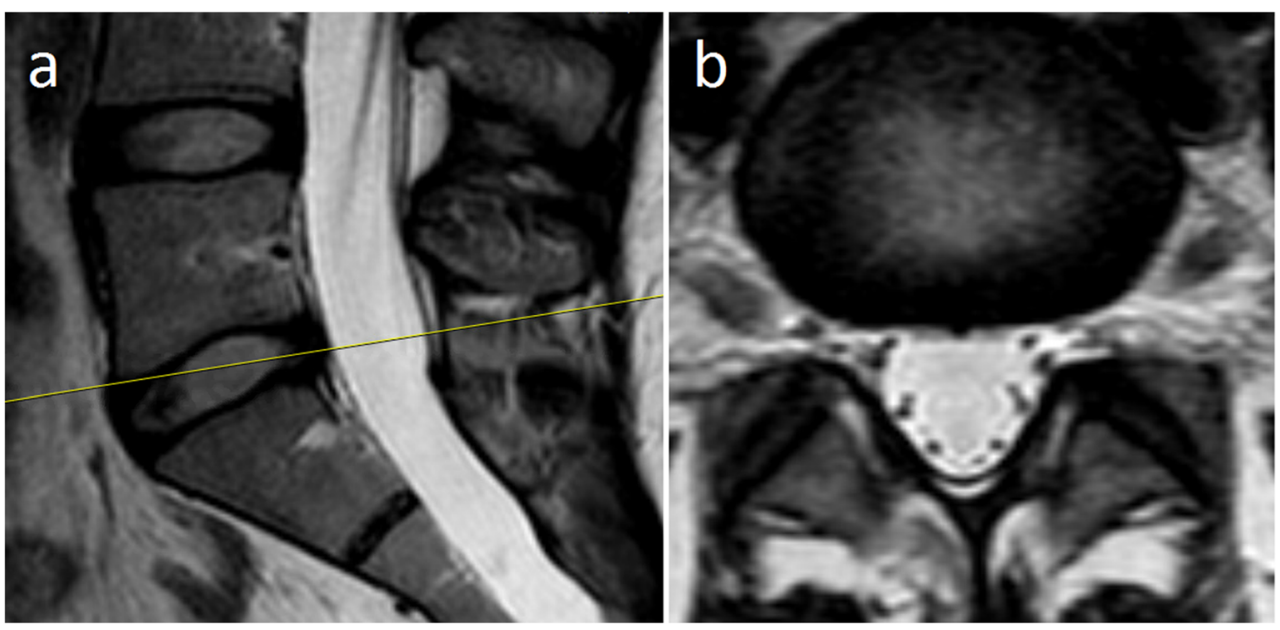


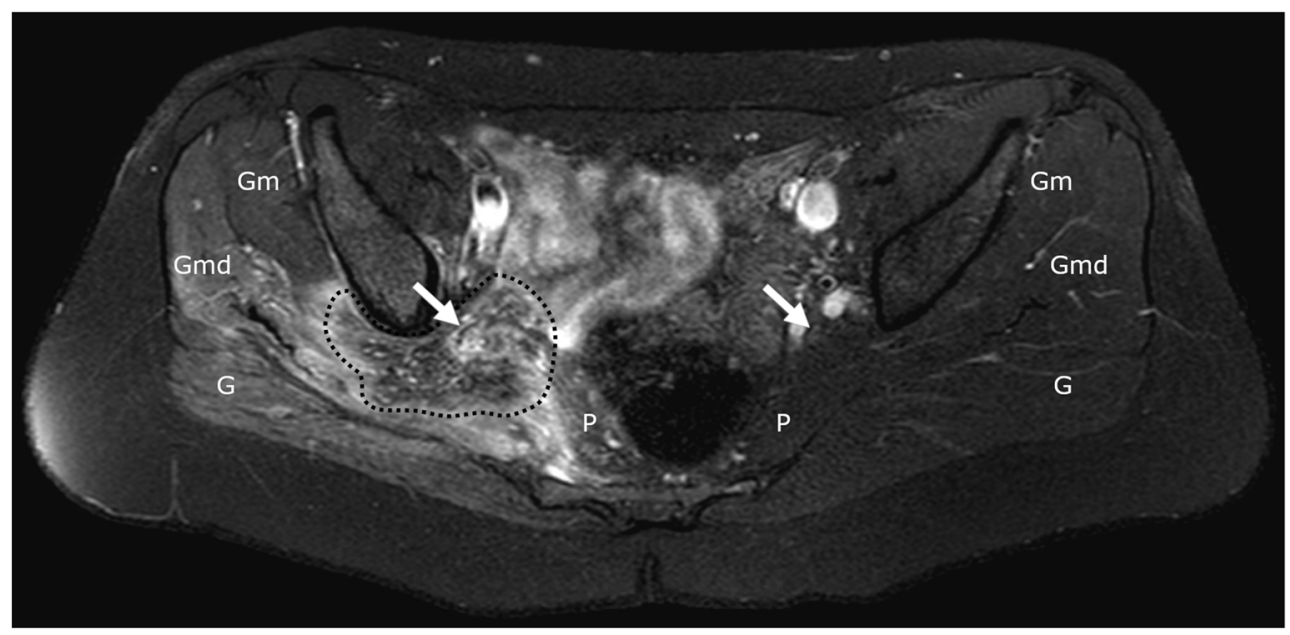

Fig. 3 Fat suppressed axial T2-weighted MRI at the level of the greater sciatic foramen shows the endometriotic mass with spiculated edges (dotted line), which is hypointense with foci of hyperintensity, traversing the foramen and surrounding the right sciatic nerve (white arrow) which is

The patient, her obstetrician, and neurologist agreed to postpone surgery and use this combination of therapies for 6 to 12 months, while monitoring symptoms and re-imaging if necessary. At a 3-month follow-up, the patient's pain was maintained at a mild level and she reported that her hip felt "great." While there were no adverse events related to the conservative course of care, it is possible that the patient could experience worsening of symptoms or motor deficits and require surgery.

\section{Discussion}

The chiropractic assessment contrasted a severe clinical picture with relatively normal lumbar imaging, which prompted further investigation. Apart from providing conservative care, the role of a chiropractor may include reviewing prior records, visibly enlarged and hyperintense compared to the left sciatic nerve (white arrow). Right-sided muscles show T2 hyperintensity consistent with early atrophy of the gluteus medius (Gmd), gluteus maximus $(\mathrm{G})$, piriformis $(\mathrm{P})$, and gluteus minimus $(\mathrm{Gm})$

providing a thorough examination, ordering imaging, referring to other providers, helping formulate a differential diagnosis, and devising a treatment plan [12].

Coordinated co-management of this case resulted in a relatively short diagnostic interval of 14 months. Early diagnosis in sciatic endometriosis is important as it provides a longer therapeutic window for conservative care, allows for time to develop a treatment plan, and limits the likelihood of ongoing nerve damage [3].

The patient's symptoms resulted from compression and inflammation of the lumbosacral plexus and neural elements traversing the greater sciatic foramen. Distal lower extremity neurologic deficits related to involvement of the L5 and S1 plexus components, while gluteal denervation could also be explained by damage to smaller plexus branches in the greater sciatic foramen, such as the superior gluteal nerve. Symptom provocation by hip internal rotation and adduction likely
Fig. 4 STIR MRI at the greater sciatic foramen showing hyperintense signal of the gluteus medius $(\mathrm{Gmd})$ and maximus $(\mathrm{G})$ and piriformis $(\mathrm{P})$ consistent with early denervation changes, hypointense endometrial mass with foci of hyperintensity (dotted line) adjacent to the lumbosacral plexus and occupying the greater sciatic foramen, and right sciatic nerve hyperintensity and enlargement (black arrowheads). Left sciatic nerve (white arrowheads) for comparison. The cranial portion of the endometrial implant surrounds the superior gluteal neurovascular bundle subjacent to the sacroiliac joint

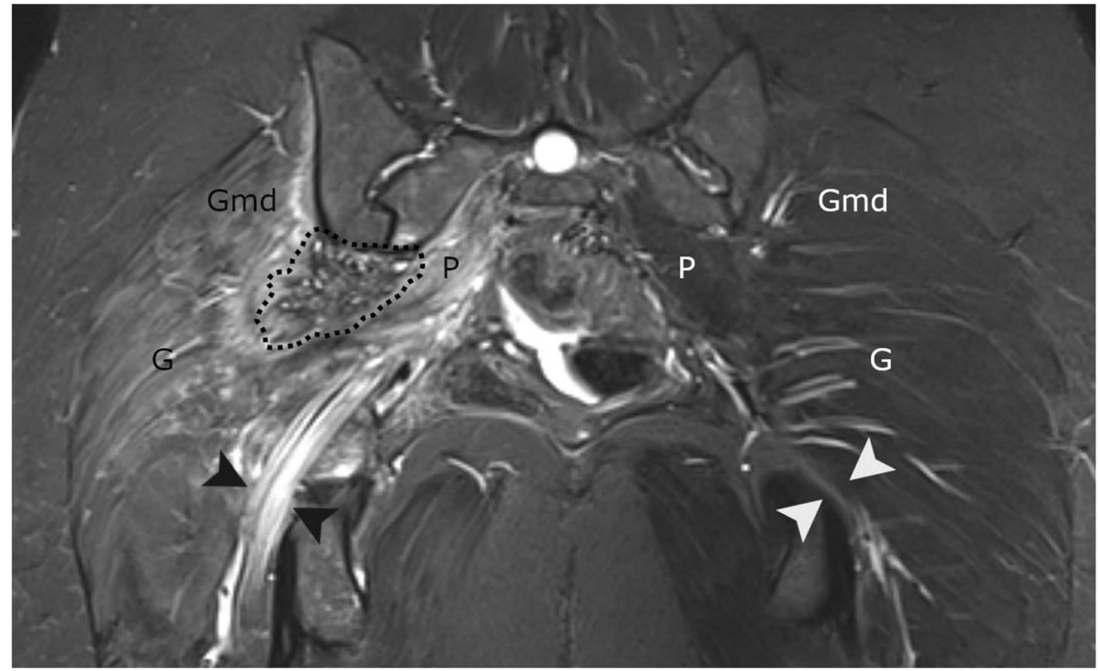


Fig. 5 Coronal T1-weighted MRI with intravenous gadolinium contrast, showing right-sided enhancement of the superior gluteal nerve in its course from the lumbosacral plexus to the suprapiriform foramen (arrowheads), enlargement and enhancement of the S1 nerve root (arrow), an enhancing endometrial mass (dotted line), and an atrophic right obturator internus $(\mathrm{OI})$

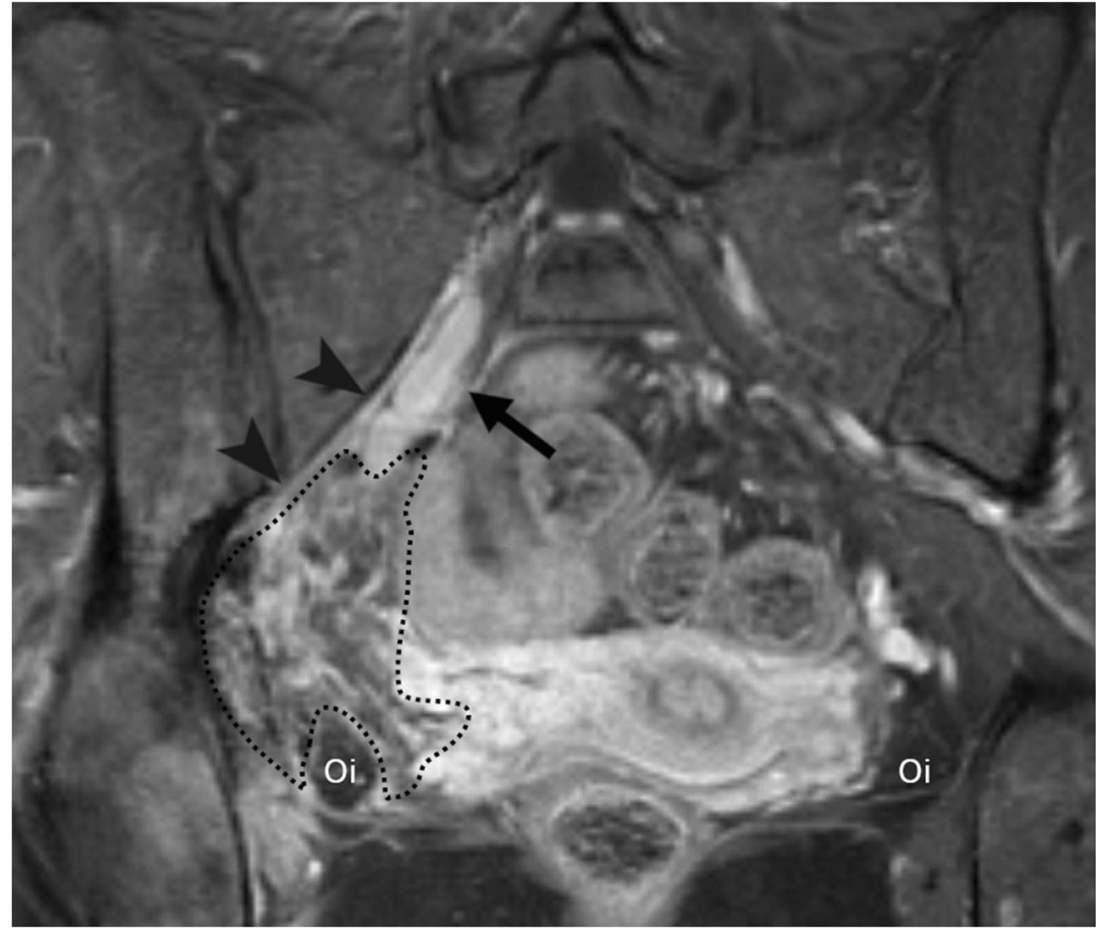

resulted from a mass effect of the endometrial implant in the greater sciatic foramen, which reduced the potential space for nerve mobility.

Prior cases have reported endometrial tissue at the greater sciatic foramen or its bony border, the sciatic notch of the ilium $[2,6,4]$. The greater sciatic foramen is a passageway for structures entering and exiting the pelvic cavity and includes the piriformis muscle and structures adjacent to it (Fig. 7). The suprapiriform foramen contains the superior gluteal neurovascular bundle, while the infrapiriform foramen contains the sciatic nerve, inferior gluteal neurovascular bundle, posterior femoral cutaneous nerve, pudendal nerve, internal pudendal vessels, and nerves to the obturator internus and quadratus femoris. In our case, in addition to involvement of the superior gluteal nerve, there was a possible entrapment of the inferior gluteal nerve and nerve to the obturator internus based on imaging signs of denervation of the gluteus maximus and obturator internus.

Treatment of sciatic endometriosis is patient-specific. Some patients respond well to hormonal therapy and do not require surgery $[5,13]$. However, patients with severe weakness such
Fig. 6 Photomicrograph showing an endometrial gland lined by columnar epithelium (arrowheads) and endometrial stroma $(*)$ with associated hemorrhage. Red blood cells are visible in the top-right lumen of the gland. Hematoxylin and eosin stain, original magnification $\times 20$

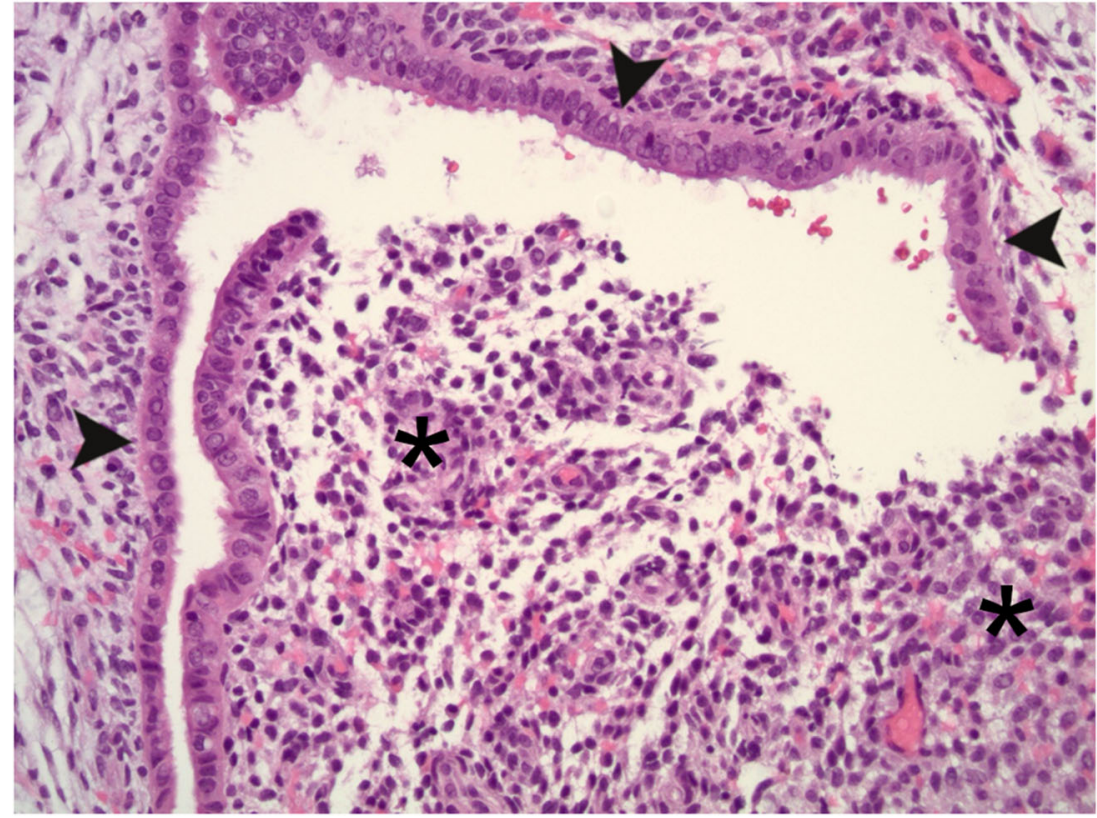




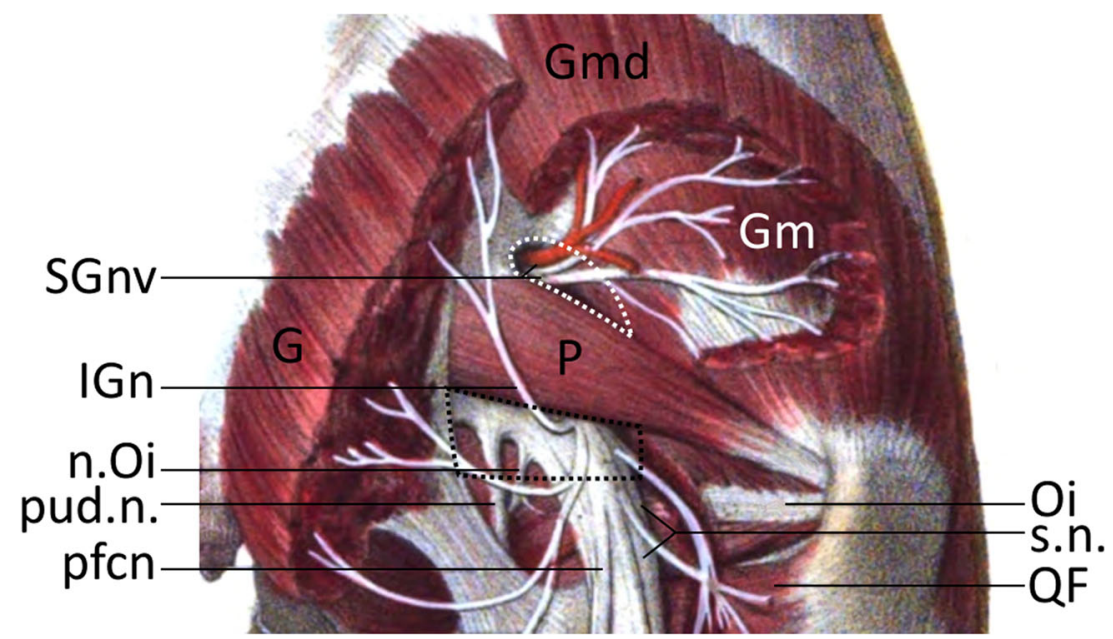

Fig. 7 Neurovascular structures emerging from the pelvis in relation to the piriformis $(\mathrm{P})$. The suprapiriform foramen (white dotted line) contains the superior gluteal neurovascular bundle (SGnv). The infrapiriform foramen (black dotted line) contains: The inferior gluteal nerve (IGn, vessels not shown), nerve to the obturator internus (n. Oi), pudendal nerve (pud. n.), posterior femoral cutaneous nerve (pfen), and sciatic nerve (s.n.). Also shown are the gluteus maximus (G), medius (Gmd), and minimus $(\mathrm{Gm})$, obturator internus $(\mathrm{Oi})$, and quadratus femoris $(\mathrm{QF})$. Image modified from Hirschfeld \& Léveillé's Névrologie et esthésiologie (1866), which is public domain as foot drop are typically recommended surgical excision of the endometrial tissue from the lumbosacral plexus $[4,14]$. Patients who initially benefit from hormone therapy may ultimately discontinue this treatment due to unwanted side effects, and then proceed to surgery [7]. One prospective study found that complete surgical removal of endometrial lesions causing lumbosacral plexopathy was successful in reducing pain and improving function; however, there was typically a long recovery period of 3 years, requiring intensive physical therapy [14].

In our case, MRI showed signs of intraneural endometriosis including abnormally thickened, $\mathrm{T} 2$ hyperintense, and gadolinium-enhancing S1 nerve root and sciatic nerve [1]. The presence of intraneural endometriosis increases the complexity of a potential surgery, which would require excising nerve tissue to completely remove the endometrial tissue [14].

The site of this case report, University Hospitals Health Systems (UH), includes over 1000 physicians in 73 clinical locations [15]. As of 2021, UH employs 5 full-time chiropractic physicians who routinely provide care for neuromusculoskeletal disorders, receive and provide referrals to specialist and primary care physicians, and chart patients' care in the UH-wide ambulatory EMR. In this case, the chiropractic physician conducted a thorough physical examination, and used the shared EMR to review diagnostic images, order additional imaging, and refer to appropriate specialists. This workup led to a revision of the patient's diagnosis and improved outcome, and provides an example of the value of integrating chiropractic physicians into a hospital system.

According to the 2020 survey from the National Board of Chiropractic Examiners (NBCE) including 2,309 respondents, only $5 \%$ of US chiropractic physicians have healthcare privileges to admit or treat patients in hospitals [16]. Similarly, in the 2015 survey from the American Chiropractic Association, only 50 of
1,142 respondents $(4 \%)$ reported working in a medical facility [17]. One notable exception is the Veterans Health Administration (VHA) which began hiring chiropractic physicians in 2004, and as of 2015 offered chiropractic services in 65 centers or hospitals [18]. Both the VA and UH are rather unique in their integration of chiropractic physicians into a hospital network.

Although management of this patient resulted in fasterthan-average diagnosis, it is possible that care would have been managed in a similar manner at an outside, nonintegrative facility. Although MRI and transvaginal ultrasound did not identify intra-peritoneal lesions, it is possible that small lesions could have been found using laparoscopy.

\section{Conclusions}

Endometriosis is a rare cause of sciatica that may be initially misdiagnosed as a lumbar spine disorder. Clinicians may recognize it by conducting a thorough evaluation, correlating clinical features with imaging, and recognizing atypical symptoms such as multi-segmental neurological involvement and a cyclical pain pattern. Providers that treat musculoskeletal and pain disorders should be aware of this condition and its time-dependent management. This case provides an example in which the placement of a chiropractor within an integrative hospital network facilitated coordination of care for a complex and rare disorder.

\section{Patient perspective}

The journey to find a cause for my pain and discomfort felt long and disorganized until I met with my chiropractor. It was 
then that I felt someone was really listening to me and looking for answers that may have not been the obvious choices. The progression of testing and diagnostics really took off after the imaging was done that she ordered. I still have a ways to go before this is behind me but I am so appreciative of everything everyone has done to help me get there.

\section{Availability of Data Not applicable.}

Code Availability Not applicable.

Author Contribution All authors RT, SP, KL, JS, and JD conceived of the case report, drafted, critically revised it, provided intellectual content, and approved of the final version to be published. SP and JS were responsible for data acquisition. RT, SP, KL, JD, and JS were responsible for data interpretation.

\section{Declarations}

Ethics Approval All procedures performed in studies involving human participants were in accordance with the ethical standards of the institutional and/or national research committee and with the 1964 Helsinki declaration and its later amendments or comparable ethical standards. This case report was declared Not Human Subjects Research by the University Hospitals Institutional Review Board.

\section{Consent to Participate Not applicable.}

Consent for publication The patient gave written consent for this article to be published.

Conflict of Interest Dr. Trager reports he has received book royalties as the author of two texts on the topic of sciatica.

Open Access This article is licensed under a Creative Commons Attribution 4.0 International License, which permits use, sharing, adaptation, distribution and reproduction in any medium or format, as long as you give appropriate credit to the original author(s) and the source, provide a link to the Creative Commons licence, and indicate if changes were made. The images or other third party material in this article are included in the article's Creative Commons licence, unless indicated otherwise in a credit line to the material. If material is not included in the article's Creative Commons licence and your intended use is not permitted by statutory regulation or exceeds the permitted use, you will need to obtain permission directly from the copyright holder. To view a copy of this licence, visit http://creativecommons.org/licenses/by/4.0/.

\section{References}

1. Lomoro P, Simonetti I, Nanni A, Cassone R, Di Pietto F, Vinci G, et al. Extrapelvic sciatic nerve endometriosis, the role of magnetic resonance imaging: case report and systematic review. J Comput Assist Tomogr. 2019;43(6):976-80.

2. Siquara De Sousa AC, Capek S, Amrami KK, Spinner RJ. Neural involvement in endometriosis: review of anatomic distribution and mechanisms. Clin Anat. 2015;28(8):1029-38.

3. Chen S. Chronic Sciatica Induced by Endometriosis. J Anesth Perioperat Med. 2018;5(6):325.

4. Floyd JR, Keeler ER, Euscher ED, McCutcheon IE. Cyclic sciatica from extrapelvic endometriosis affecting the sciatic nerve: Case report. J Neurosurg Spine. 2011;14(2):281-9.

5. de Sousa ACS, Capek S, Howe BM, Jentoft ME, Amrami KK, Spinner RJ. Magnetic resonance imaging evidence for perineural spread of endometriosis to the lumbosacral plexus: report of 2 cases. Neurosurg Focus. 2015;39(3):E15.

6. Salazar-Grueso E, Roos R. Sciatic endometriosis: a treatable sensorimotor mononeuropathy. Neurology. 1986;36(10):1360.

7. Uppal J, Sobotka S, Jenkins AL III. Cyclic sciatica and back pain responds to treatment of underlying endometriosis: case illustration. World Neurosurg. 2017;97:760. e1-3.

8. Rutkove SB, Sax TW. Lumbosacral plexopathies. Neuromuscular disorders in clinical practice: Springer; 2014. p. 1063-71.

9. Laporte C, Albert J-D, Duvauferrier R, Bertaud V, Gouillou M, Guillin R. MRI investigation of radiating pain in the lower limbs: value of an additional sequence dedicated to the lumbosacral plexus and pelvic girdle. Am J Roentgenol. 2014;203(6):1280-5.

10. Reddy S, Porter D, Patton J, Al-Nafussi A, Beggs I. Endometriosis of the superior gluteal nerve. Skelet Radiol. 2007;36(9):879-83.

11. Saar TD, Pacquée S, Conrad DH, Sarofim M, De Rosnay P, Rosen $\mathrm{D}$, et al. Endometriosis involving the sciatic nerve: a case report of isolated endometriosis of the sciatic nerve and review of the literature. Gynecol Minim Invasive Ther. 2018;7(2):81.

12. Souza TA. Differential diagnosis and management for the chiropractor: protocols and algorithms: Jones \& Bartlett Publishers; 2009.

13. Yekeler E, Kumbasar B, Tunaci A, Barman A, Bengisu E, Yavuz E, et al. Cyclic sciatica caused by infiltrative endometriosis: MRI findings. Skelet Radiol. 2004;33(3):165-8.

14. Possover M. Five-year follow-up after laparoscopic large nerve resection for deep infiltrating sciatic nerve endometriosis. J Minim Invasive Gynecol. 2017;24(5):822-6.

15. Find a Location. University Hospitals. https://www.uhhospitals. org/locations. Accessed 8/11/2020.

16. Himelfarb I, Hyland J, Ouzts N, Russell M, Sterling T, Johnson C, et al. National Board of Chiropractic Examiners: Practice Analysis of Chiropractic 2020 [Internet]. Greeley, CO: NBCE; 2020 [cited 2020 Nov 7]. Available from: https://www.nbce.org/practiceanalysis-of-chiropractic-2020/.

17. Bronston LJ, Austin-McClellan LE, Lisi AJ, Donovan KC, Engle WW. A survey of American Chiropractic Association members' experiences, attitudes, and perceptions of practice in integrated health care settings. J Chiropract Med. 2015;14(4):227-39.

18. Lisi AJ, Brandt CA. Trends in the use and characteristics of chiropractic services in the Department of Veterans Affairs. J Manip Physiol Ther. 2016;39(5):381-6.

Publisher's Note Springer Nature remains neutral with regard to jurisdictional claims in published maps and institutional affiliations. 